\title{
Magnetic field generation in an accretion disk of close binary star
}

\author{
Dmitry V. Bisikalo and Andrey G. Zhilkin \\ Institute of Astronomy of the RAS, \\ 48 Pyatnitskaya str., Moscow, Russia, \\ email: bisikalo@inasan.ru
}

\begin{abstract}
Using results of 3D MHD numerical simulations we investigate the generation of the magnetic field in an accretion disk of a close binary star. Analysis of the numerical simulations shows that the magnetic field, which is mainly toroidal, is intensively generated in the accretion disk. In the disk, we can distinguish three regions: inner region of the intensive toroidal field generation due to the differential rotation; region of the current sheets; and outer region of the magnetic field dissipation. In the outer regions of the accretion disk of a magnetic close binary system two types of the dynamo may exist. One of them is the laminar dynamo that occurs as a result of the nonaxisymmetric motions. The other type is the turbulent $\alpha \omega$-dynamo. Numerical simulations show that during the disk lifetime the dynamo effect in the outer region of the disk can be significant.
\end{abstract}

Keywords. Accretion disks, binaries: close, MHD, dynamo.

\section{Introduction}

In acretion disks that form in close binary systems, the proper magnetic field of the compact object can be amplified by the differential rotation, radial motions and dynamo. The field is reduced by diffusion, turbulent dissipation and magnetic buoyancy. The action of these effects results in the formation of a magnetic field with a rather complex structure, since different effects can dominate in different regions of the disk.

We can pick the following main processes that determine the structure of the field in the accretion disk. In the inner regions of the disk the main process is the generation of toroidal magnetic field due to differential rotation (Campbell 1997). The structure of the generated field is determined by the rotation law of the disk. However the effects concerned with the existence of poloidal velocity in the disk, redistributing the field over the disk, can become significant here (Bisnovatyi-Kogan \& Ruzmaikin 1974, BisnovatyiKogan \& Lovelace 2007, Rothstein \& Lovelace 2008). The magnetic field can interact with waves, occurring in the inner regions of the disk (Bisikalo et al. 2004, Bisikalo et al. 2005), which leads to quasi-periodic oscillations of the accretion rate (Zhilkin \& Bisikalo 2010).

In the outer regions of the disk magnetic field can be partly amplified by dynamo. In the accretion disks of close binary systems two types of dynamo can work: laminar dynamo (due to non-axisymmetric motions, Braginsky 1964) and turbulent $\alpha \omega$-dynamo (Parker 1982). The dynamo-generation of magnetic field was studied, for example, in (Parker 1971, Pudritz 1981, Campbell 1997). In this work, using results of 3D MHD numerical simulations, we investigate the generation of magnetic field in the accretion disk of a close binary star. 


\section{Standard approach}

The evolution of magnetic field in an accretion disk is determined by the induction equation

$$
\frac{\partial \mathbf{B}}{\partial t}=\nabla \times\left[\mathbf{v} \times \mathbf{B}-\eta_{\mathrm{OD}}(\nabla \times \mathbf{B})\right],
$$

where $\mathbf{B}$ is the vector of magnetic induction; $\mathbf{v}$ - velocity; $\eta_{\mathrm{OD}}$ is the coefficient of $\mathrm{Ohm}$ diffusion. The total magnetic field in the disk can be presented as a sum of the proper magnetic field of the accretor $\mathbf{B}_{*}$ and the magnetic field $\mathbf{b}$, generated by currents in the disk plasma: $\mathbf{B}=\mathbf{B}_{*}+\mathbf{b}$. Since the field $\mathbf{B}_{*}$ is generated by currents inside the accretor, in the disk it should satisfy the potentiality condition: $\nabla \times \mathbf{B}_{*}=0$. In the general case the spin rotation of the accretor is asynchronous. Here we are considering the case when the spin rotation axis of the accretor coincides with the axis of the orbital rotation. Since the spin rotation of the accretor is asynchronous, its magnetic field is non-stationary.

Variations of $\mathbf{B}_{*}$ with time can be described by the following equation:

$$
\frac{\partial \mathbf{B}_{*}}{\partial t}=\nabla \times\left(\mathbf{v}_{*} \times \mathbf{B}_{*}\right),
$$

where $\mathbf{v}_{*}$ is the velocity of accretor's field lines.

Using those relations we find that the proper magnetic field of the disk satisfies the equation:

$$
\frac{\partial \mathbf{b}}{\partial t}=\nabla \times(\mathbf{v} \times \mathbf{b})+\nabla \times\left[\left(\mathbf{v}-\mathbf{v}_{*}\right) \times \mathbf{b}\right]-\nabla \times\left[\eta_{\mathrm{OD}}(\nabla \times \mathbf{b})\right] .
$$

Since the flow in the accretion disk is turbulent, the velocity $\mathbf{v}$ and magnetic field $\mathbf{b}$ can be presented as sums of the mean values $\langle\mathbf{v}\rangle,\langle\mathbf{b}\rangle$ and their fluctuations $\delta \mathbf{v}, \delta \mathbf{b}$.

For turbulent pulsations the mean value is:

$$
\langle\delta \mathbf{v} \times \delta \mathbf{b}\rangle=\alpha\langle\mathbf{b}\rangle-\beta \nabla \times\langle\mathbf{b}\rangle,
$$

where $\alpha$ is the axisymmetric function of coordinates, concerned with the helicity of the flow, and $\beta$ is the coefficient of magnetic viscosity, conditioned by turbulence. Thus, the induction equation for the averaged values can be re-written as follows:

$$
\frac{\partial\langle\mathbf{b}\rangle}{\partial t}=\nabla \times\left[\langle\mathbf{v}\rangle \times\langle\mathbf{b}\rangle+\alpha\langle\mathbf{v}\rangle+\left(\langle\mathbf{v}\rangle-\mathbf{v}_{*}\right) \times\langle\mathbf{b}\rangle-\eta(\nabla \times\langle\mathbf{b}\rangle)\right],
$$

where $\eta=\eta_{\mathrm{OD}}+\beta$ is the total diffusion coefficient of magnetic field.

To reveal the main features of the field generation process in an accretion disk let us consider a simplified model where the axis of the accretor's magnetic dipole coincides with its spin rotation axis. In this case we can use an axisymmetric approach to describe the field, averaged over turbulent pulsations. In the conditions of the accretion disk the induction equation $(2.5)$ can be significantly simplified. Since in the disk $\left|v_{r}\right|,\left|v_{z}\right| \ll$ $\left|v_{\varphi}\right|$, we can equate the poloidal velocity to zero. Besides, we can neglect insignificant terms, responsible for the $\alpha^{2}$-dynamo effect. In the diffusion terms we can neglect radial derivatives in comparison to the vertical derivatives, since their ratio is $z / r \ll 1$. After those simplifications we obtain the following system:

$$
\frac{\partial A}{\partial t}=\alpha B_{\varphi}+\eta \frac{\partial^{2} A}{\partial z^{2}}, \quad \frac{\partial B_{\varphi}}{\partial t}=\frac{z}{r} g B_{0}-g \frac{\partial A}{\partial z}+\eta \frac{\partial^{2} B_{\varphi}}{\partial z^{2}} .
$$

Here $A$ is the azimuthal component of the potential of the poloidal magnetic field of plasma $\mathbf{b}_{p}$ and $g=r \partial \omega / \partial r$ is the measure of the differential rotation; $\omega$ is the angular velocity of the disk rotation; $B_{0}=B_{\mathrm{a}}\left(R_{\mathrm{a}} / r\right)^{3}$ - specific value of the induction of the accretor's proper magnetic field in the disk; $R_{\mathrm{a}}$ - stellar radius; $B_{\mathrm{a}}$ is the magnetic field 


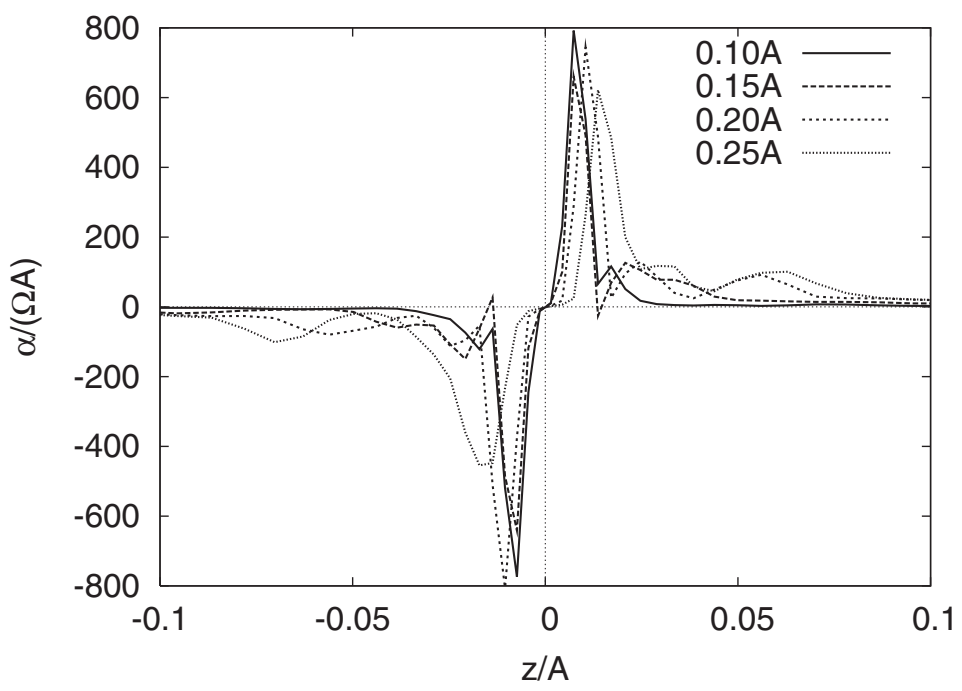

Figure 1. Distribution of the mean helicity $\alpha$ in the accretion disk at different distances from the accretor.

induction on the surface of the accretor. These equations have a simple physical sense. The first equation in (2.6) describes the evolution of poloidal magnetic field in the disk. The first term in the right-hand side determines the generation of the poloidal field from the toroidal due to the dynamo effect, while the second term is responsible for the dissipation of the poloidal field. The second equation in (2.6) describes the evolution of toroidal magnetic field field in the disk. The first and second terms in the right-hand side of this equation determine the generation of the toroidal field from the accretor's proper field and previously generated poloidal field due to the differential rotation of the disk. The last term in the right-hand side describes the dissipation of the toroidal magnetic field.

In the inner regions of the disk the dynamo effect is weak and the field is generated due to the differential rotation in the disk. In this case in the first equation of (2.6) we can neglect the term $\alpha B_{\varphi}$. This results in $A=0$ and, hence, only the toroidal field is generated. The second equation in (2.6) now is as follows:

$$
\frac{\partial B_{\varphi}}{\partial t}=\frac{z}{r} g B_{0}+\eta \frac{\partial^{2} B_{\varphi}}{\partial z^{2}}
$$

In the outer regions of the disk the field can be amplified due to dynamo. The stationary distribution of the field is described by the equation:

$$
\frac{\partial^{3} B_{\varphi}}{\partial z^{3}}+\frac{\alpha g}{\eta^{2}} B_{\varphi}+\frac{g B_{0}}{\eta r}=0 .
$$

This equation is invariant with respect to the transformation $z \rightarrow-z$ and simultaneous substitution $B_{\varphi}(z) \rightarrow-B_{\varphi}(-z)$. This means that it describes the generation of the dipole (anti-symmetric) component of the magnetic field where $B_{\varphi}(-z)=-B_{\varphi}(z)$. However, at long distances from the star the quadrupole (symmetric) component $\left(B_{\varphi}(-z)=B_{\varphi}(z)\right)$ of the field can dominate (Ruzmaikin, Sokoloff \& Shukurov 1988). 


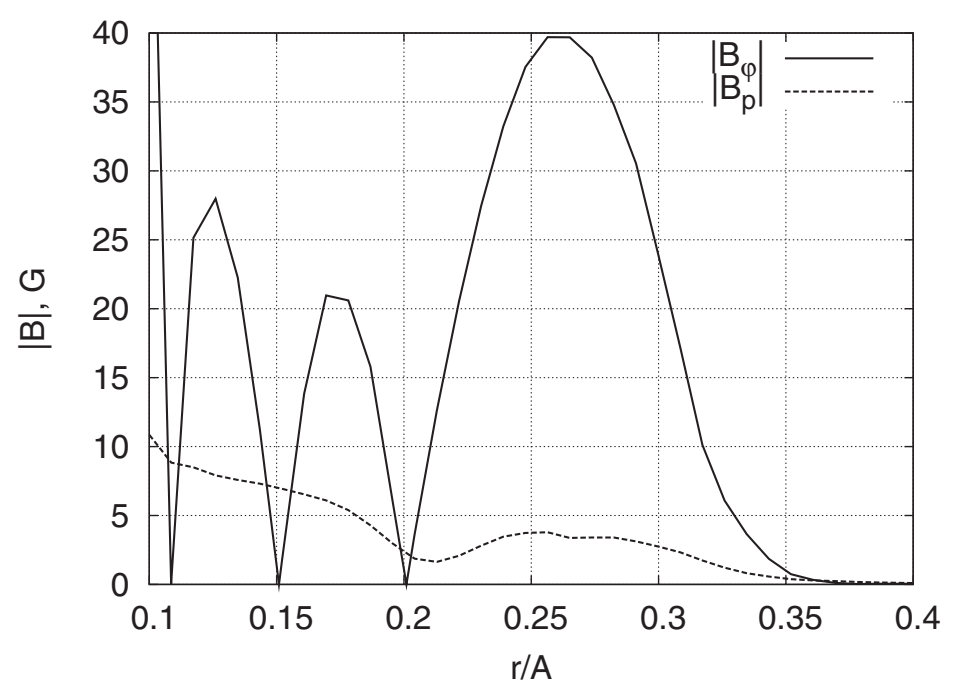

Figure 2. Azimuthally averaged absolute values of toroidal $B_{\varphi}$ and poloidal $B_{p}$ magnetic field in the equatorial plane of the accretion disk.

\section{Features of dynamo in close binary systems}

Dynamo-generation of magnetic field in accretion disks of close binary systems has its specific features. Let us describe the main of them.

1. The specific time of the accretor's (white dwarf or neutron star) magnetic field decay is much longer than the specific lifetime of the accretion disk. This makes the steady generation of the toroidal field in the disk due to the differential rotation ( $\omega$-dynamo) possible. This effect dominates in the inner regions of the disk.

2. Both the types of dynamo (turbulent and laminar) work. The first one is possible since the flow in the disk is turbulent. The second one works since accretion disks in close binaries are elliptical and, hence, there is no axial symmetry of the flow. In this case the velocity $\mathbf{v}$ and magnetic field $\mathbf{b}$ can also be presented as sums of azimuthally averaged values $\langle\mathbf{v}\rangle,\langle\mathbf{b}\rangle$ and fluctuations $\delta \mathbf{v}, \delta \mathbf{b}$. Here:

$$
\langle\delta \mathbf{v} \times \delta \mathbf{b}\rangle=\alpha\langle b\rangle \mathbf{n}_{\varphi},
$$

where $\mathbf{n}_{\varphi}$ is the unit azimuthal vector. If the field is almost toroidal, these formulae for turbulent and laminar dynamos (in $\alpha$-effect) give very close results.

In Fig. 1 we show vertical distributions of $\alpha$ for non-azimuthal velocity fluctuations in the accretion disk at different distances from the accretor. Here A is the binary separation. The distributions were obtained as a result of the 3D numerical MHD simulations. Figure shows that the helicity has the mirror anti-symmetry with respect to the disk plane which is necessary condition for dynamo to work (Ruzmaikin, Sokoloff \& Shukurov 1988). Probably, for the turbulent velocity fluctuations, the profiles should be similar.

3. The mean helicity of the flow in accretion disks has no mirror symmetry. The number of the left-hand vortices is not equal to the number of the right-hand vortices. The additional twist comes from the Coriolis force occurring due to the Keplerian rotation of the disk. In the close binaries the additional Coriolis force occurs as a result of the orbital rotation of the stellar components. This effect influences the action of both the turbulent and laminar dynamos. 

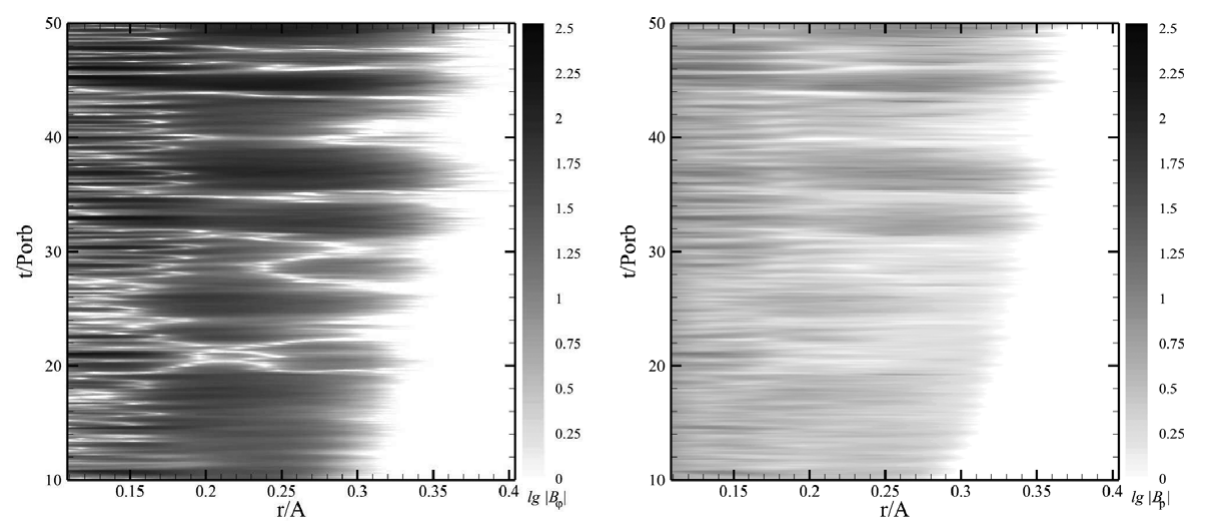

Figure 3. Radial distributions of azimuthally averaged absolute values of toroidal $B_{\varphi}$ (left panel) and poloidal $B_{\mathrm{p}}$ (right panel) magnetic field depending on time.

\section{Results and Discussion}

Analyzing results of 3D numerical calculations we can conclude that in the disk we can clearly distinguish three regions: inner region of the intensive generation of the toroidal field due to the differential rotation; current sheet region; and outer region of the field dissipation. In Fig. 2 we show azimuthally averaged absolute values of toroidal $B_{\varphi}$ and poloidal $B_{p}$ magnetic field in the equatorial plane of the accretion disk. Between the distances of $0.1 A$ to $0.2 A$ from the accretor the current sheets form in the disk. In the shown graph, the locations of the current sheets correspond to the points where the induction of the toroidal field becomes equal to zero. In the current sheet zone the absolute value of the toroidal field is 3-4 times larger than that of the poloidal field. In the outer region of the disk, between $0.2 A$ to $0.35 A$, the toroidal magnetic field is an order of magnitude stronger than the poloidal field. However, Fig. 2 shows that in this zone weak poloidal field is generated. The current sheet forms somewhere in the outer boundary of the inner zone or even inside this zone. The variation of the sign of $B_{\varphi}$ is concerned with the variation of the rotation law in the disk. Near the star a transition region is formed where matter actively loses angular momentum. In this zone the angular velocity of gas decreases from the Keplerian values to the velocity of the accretor's field lines rotation. The variations of the rotation law influences the generation of toroidal magnetic field.

Fig. 2 shows that in the outer region $(0.2 A \leqslant r \leqslant 0.35 A)$ of the disk, indeed, the poloidal component of the field is generated, since in this region it has a well seen local maximum. By the moment $t \approx 11 \mathrm{P}_{\text {orb }}$, corresponding to the distributions shown in Figure, the magnetic field is still mostly toroidal. However, we can expect that at longer time intervals the poloidal component can significantly grow and become equal in its specific value to the toroidal component.

The evolution of the radial distributions of azimuthally averaged absolute values of the toroidal and poloidal magnetic fields at longer time intervals of 10 to 50 orbital periods is shown in Fig.3. The value of the field is given in Gauss and shown as greyscale. Figure clearly shows the formation and subsequent redistribution of the current sheets by zigzag light lines. Analysis of Figure allows us to conclude that the period of quasiperiodic oscillations of the magnetic field value in the disk is approximately one orbital period. However against a background of these quasi-periodic oscillations the toroidal and poloidal fields gradually grow. At $t=10 \mathrm{P}_{\text {orb }}$ the ratio of specific values of toroidal and poloidal fields in the outer zone is approximately equal to 10 (see Fig.2). By the moment of $t=50 \mathrm{P}_{\text {orb }}$ this ratio becomes equal to 8 . When extrapolating these data 
to longer time intervals, we can conclude that the values of the toroidal and poloidal fields become equal in the outer regions of the disk in approximately 200 orbital periods. This specific time is comparable with the specific lifetime of the disks in the considered systems. Therefore, the obtained estimate shows that during the disk lifetime the dynamo effect in the outer region of the disk can be significant.

\section{Acknowledgements}

This work was supported by the Basic Research Program of the Presidium of the Russian Academy of Sciences, Russian Foundation for Basic Research (projects 11-02-00076, 12-02-00047), Federal Targeted Program "Science and Science Education for Innovation in Russia 2009-2013".

\section{References}

Campbell C. G. 1997, Magnetohydrodynamics in binary stars (Dordrecht/Boston/London: Kluwer Academic Publishers)

Bisnovatyi-Kogan G. S. \& Ruzmaikin A. A. 1975, Astrophys. and Space Sci., 28, 45

Bisnovatyi-Kogan G. S. \& Lovelace R. V. E.. 2007, ApJ, 667, L167

Rothstein D. M. \& Lovelace R. V. E.. 2008, ApJ, 677, 1221

Bisikalo D. V., Boyarchuk A. A., Kaigorodov P. V., \& Kuznetsov O. A. 2004, Astron. Rep., 48, 449

Bisikalo D. V., Boyarchuk A. A., Kaigorodov P. V., \& Kuznetsov O. A. 2005, Astron. Rep., 49, 701

Zhilkin A. G. \& Bisikalo D. V. 2010, Advances in Space Research, 45, 437

Braginsky S. I. 1964, Sov. Phys. JETP, 20, 1462

Parker E. 1982, Cosmical Magnetic Fields (Claredon: Oxford)

Parker E. N. 1971, ApJ, 163, 255

Pudritz R. E. 1981, Mon. Not. R. Astron. Soc., 195, 897

Ruzmaikin A. A., Sokoloff D. D., \& Shukurov A. M. 1988, Magnetic Fields of Galaxies (Kluwer:Dordrecht) 